\title{
Pediatric Stainless-Steel Crown Cementation Finite Element Study
}

\author{
Ahmed S. Waly ${ }^{1,2} \quad$ Yasser R. Souror ${ }^{1,3, \odot}$ \\ ${ }^{1}$ Department of Pediatric Dentistry and Dental Public Heath, Faculty \\ of Dental Medicine, Al-Azhar University Assuit Branch, Egypt \\ ${ }^{2}$ Department of Restorative Dental Sciences, Alfarabi Colleges for \\ Dentistry and Nursing, Jeddah, Saudi Arabia \\ ${ }^{3}$ Division of Pediatric Dentistry, Faculty of Dentistry, Batterjee \\ Medical College for Science and Technology, Jeddah, Saudi Arabia \\ ${ }^{4}$ Crown and Bridge Department, Faculty of Dental Medicine, Al \\ Azhar University Assiut Branch, Egypt \\ ${ }^{5}$ Department of Prosthetic Dentistry, College of Dentistry, King \\ Khalid University, Abha, Saudi Arabia \\ ${ }^{6}$ Department of Mechanical Engineering, National Research Centre, \\ Cairo Governorate, Egypt
}

Salah A. Yousief ${ }^{2,4}$

Waleed M.S. Alqahtani ${ }^{5}$

Mohamed I. El-Anwar ${ }^{6}$

Address for correspondence Ahmed S. Waly, Department of Pediatric Dentistry and Dental Public Heath, Faculty of Dental Medicine, Al-Azhar University Assuit Branch, Jeddah 23452, Egypt (e-mail: dr.waly78s@gmail.com).

Eur J Dent:2021;5:77-83

\begin{abstract}
Keywords

- Hall technique

- stainless steel crown

- finite element

- cement

- zinc phosphate

- glass ionomer
\end{abstract}

Objective To study the effect of using different cement types under pediatric stainless-steel crown (SSC) around mandibular second primary molar using three-dimensional (3D) finite element analysis.

Materials and Methods A 3D finite element model was built for pediatric mandibular molar by laser scanning of natural extracted tooth. Four types of cement (zinc phosphate, glass ionomer, resin-modified glass ionomer, and resin) of $200 \mu \mathrm{m}$ layers thickness were tested under a stainless-steel crown of 130- $\mu \mathrm{m}$ thickness. Twelve case studies were reported within this research, as the applied load of $330 \mathrm{~N}$ was tested with three angulations: vertical, oblique at $45^{\circ}$, and laterally.

Results Linear static stress analysis was performed. The resultant stresses and deformations' distribution patterns did not change with cement type, while the values were altered. All deformations and stresses were found within the normal range.

Conclusions Analysis results indicated that using stiffer cement material increases tooth structure stresses and reduces crown body stresses and deformations, while bone was nearly insensitive to cement type.

\section{Introduction}

The chrome steel crown was introduced in the 1950s and has since been regularly used in pediatric dentistry. Earlier, crown layouts were simple. Later, the precountered and pretrimmed crowns were introduced, which made the delivery of stainless-steel crowns (SSC) easier, although necessitating slight modification. ${ }^{1}$

The reasons behind SSCs popularity are excellent durability, relative inexpensiveness, and the requirement of a single dental visit. This prompt restoration can be interesting to dental professionals as it saves chair time and ensures the highest success. ${ }^{2}$ The resilience the SSC has is attributed to the chromium oxide layer, which not only makes it more durable and easy to modify but also tolerated by children. ${ }^{3}$

The physical characteristics of the SSCs got the clinician enthusiastic to accept what is known as the largest revolution in pediatric dentistry research in the last decade, the Hall technique. A Hall crown is one in which there has been no previous handpiece preparation for the primary molars and the SSC is just cemented to the tooth. This technique was developed to allow the use of preformed metal crowns without removing any tooth structure and subsequently not requiring local anesthesia. ${ }^{4}$ 
The Hall technique can be a significant addition to the numerous treatment choices for carious primary molars available to clinicians. This technique is strongly supported by encouraging outcomes, which indicate high acceptance, durability, and low-failure rates in comparison to traditional restoration procedures common to primary teeth. ${ }^{5}$

With regard to survival rate, the findings of the retrospective studies comparing traditional SSCs with Hall crowns showed a comparable and sometimes superior success rate in favor of the latter until normal shedding of primary teeth. ${ }^{6}$ From parents and children to dental professionals, everyone found the Hall crown to be highly satisfactory ${ }^{7}$

On the other hand, and as a ramification of Hall crown application, the bite rises, and potentially harmful effects of the occlusion could occur. Moreover, it could reduce the activity of masseter muscle in children. However, if the occlusion returns to normal, as it was before treatment between 15 to 30 days, children have to live with premature contact for several weeks. ${ }^{8}$

The occlusal forces on full coverage restoration were transferred to the periodontium, which can stimulate some changes in the bone and connective tissues. This force may induce tooth mobility and develop a periodontal pocket. The relationships between occlusion and the onset of loss of attachment due to inflammatory periodontal diseases cannot be ignored. ${ }^{9}$

While SSCs have a high-success rate, a significant explanation for their clinical failure is crown loss due to cementation failure. The luting cement improves the retention of crown to the prepared tooth, offers mechanical resistance to crown displacement, and even prevents fracturing under mastication force. As the luting cement adheres to the tooth surface and restoration, stability is further strengthened. ${ }^{10}$

Speaking of cementation, most published studies have been conducted to measure the shear bond strength of the cement to SSC. Although it enlightens the clinicians, it gives no idea about the biological effects of different types of cement on the tooth and alveolar bone. Hence, the objective of this study was to demonstrate the mechanical behavior of SSCs placed by the Hall technique to withstand masticatory forces when different cement types have been used and to investigate the effects on tooth structure and alveolar bone through three-dimensional (3D) finite element analysis.

To explore the mechanical properties of a prosthetic restoration with different supporting tissues, functional simulation technique is required. The finite element method (FEM) is a numerical method utilized for analyzing the framework. It is made up of a computer model or design assigned by material (s) that receive external forces and analyzed to give outcomes like deformations, stresses, and strains. ${ }^{11}$

The virtual model geometry, which is the base for building finite element model, might be as close as possible to clinical reality. The digital imaging technologies like CT, laser, probe scanning, and CAD software (s) can provide acceptable geometric (solid) model (s). Solid models of the bone, dental implants, attachments, and prosthetic crowns can be imported as 3D models' formats into FEM software. Some simplification to the virtual model may be applicable, which will not affect results obtained. Simplify the model materials like assuming homogeneous, isotropic, or linear elastic behavior, which are usually assumed without affecting results, as the analysis resulted in stresses less than yield stress. On the other hand, changing the properties of materials influence the stress distribution in the structure. The chewing forces are cyclic forces; however, they are usually simulated as static forces (extreme values). The resultant stresses judge if cyclic load should be simulated and if it exceeds a certain limit (material endurance limit). ${ }^{12}$

FEM is fit to carry out unlimited simulations without the need for animal or human tests. In dentistry, FEM provides an excellent tool for assessment of anatomical structures, and any restoration for rehabilitation to have mechanical properties and accepted load cycle testing. ${ }^{13}$

In FEM, the direction of the force exerted as well as its magnitude are readily adjusted to imitative the clinical condition. Moreover, test repetition does not affect the physical properties' tested material, which eliminates the disadvantage of interfering with the testing device on the outcomes. All these advances of FEM make it a promising technology solution not only in prosthetic dentistry and implants but also in orthodontics. ${ }^{14-17}$

\section{Materials and Methods}

A finite element model for primary second mandibular molar was developed by laser scanning for freshly extracted sound tooth due to periodontal disease after parental acceptance. The tooth geometry was acquired by using laser scanner (Geomagic Capture, 3D Systems, Cary, NC, USA). Such a scanner produced a data file containing a cloud of point coordinates (-Fig. 1). An intermediate software was required (Rhino 3.0 - McNeel Inc.; Seattle, WA, USA) to trim the newly created surfaces by the acquired points. Thereafter, the solid (closed) tooth geometry was exported to the finite element program in the STEP file format. ${ }^{18}$

On the other hand, cortical and spongy bone models were created by commercial computer-aided design softwares such as AutoDesk Inventor software version 8.0 (Autodesk Inc.; San Rafael, CA, USA). The bone geometry was simplified and simulated as two coaxial cylinders, the inner one representing the spongy bone with $14 \mathrm{~mm}$ diameter and $22 \mathrm{~mm}$ height, which fills the internal cylindrical space of the other cylinder (shell of $1 \mathrm{~mm}$ thickness) that represents cortical bone (outer diameter of $16 \mathrm{~mm}$ and its height of $24 \mathrm{~mm}$ ). ${ }^{19,20}$

A set of Boolean operations on ANSYS environment (ANSYS Inc.; Canonsburg, PA, USA) were made to finalize the model. All materials were considered isotropic, linear and elastic, which were fed to the ANSYS environment, as listed in - Table 1.

The meshing of the models' components was done by 3D brick solid element "187," which has three degrees of freedom (translation in main axes directions). ${ }^{20}$ The resulting numbers of nodes and elements are listed in - Table $\mathbf{2}$, and the meshed model components are presented as screenshots from ANSYS in - Fig. 2. 


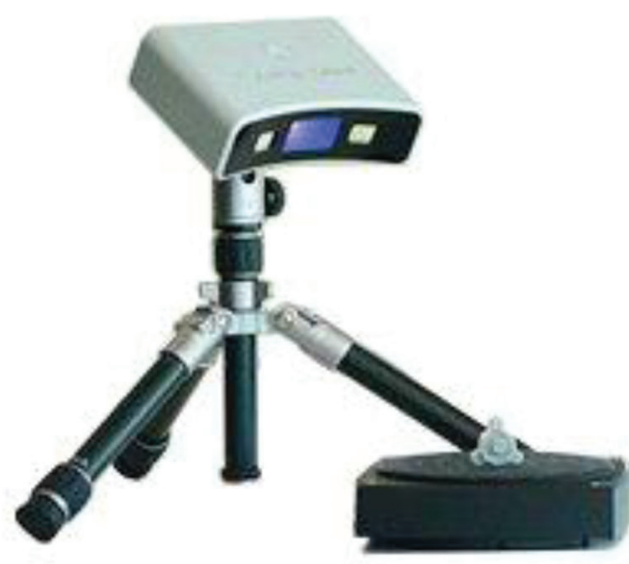

A

Fig. 1 (A) Laser scanner, (B) scanned tooth.

Table 1 Material properties of used in the finite element $\operatorname{model}(\mathrm{s})^{21}$

\begin{tabular}{|l|l|l|}
\hline Material & $\begin{array}{l}\text { Young's modulus } \\
{[\mathrm{GPa}]}\end{array}$ & Poisson's ratio \\
\hline Stainless steel & 210.0 & 0.33 \\
\hline Zinc phosphate & 22.4 & 0.35 \\
\hline (GIC) & 12.0 & 0.25 \\
\hline (RMGIC) & 4.0 & 0.30 \\
\hline Resin Cement & 7.0 & 0.27 \\
\hline Dentine & 18.6 & 0.31 \\
\hline Enamel & 84.1 & 0.30 \\
\hline Cortical bone & 13.7 & 0.30 \\
\hline Cancellous bone & 1.37 & 0.30 \\
\hline
\end{tabular}

Abbreviations: GIC, glass ionomer cement; RMGIC, resin modified glass ionomer cement.

Table 2 Mesh density of the two models' components

\begin{tabular}{|l|l|l|}
\hline \multirow{2}{*}{ Material } & \multicolumn{2}{|c|}{ Model 1 } \\
\cline { 2 - 3 } & Number of elements & Number of nodes \\
\hline Crown & 38,032 & 18,835 \\
\hline Cement & 13,272 & 6,520 \\
\hline Dentine & 50,233 & 34,626 \\
\hline Enamel & 13,166 & 7,179 \\
\hline Cortical bone & 21,661 & 11,066 \\
\hline Spongy bone & 104,591 & 74,508 \\
\hline
\end{tabular}

Three loading cases were studied for each cement material as follows: load of $330 \mathrm{~N}$ to be applied vertically, in oblique fashion, and laterally. Three points on the outer inclines of the buccal cusps and two points on the inner inclines of the lingual cusps were loaded ( $\mathbf{- F i g}$. 2 F). The model was verified against similar studies ${ }^{19,20}$ prior to extracting analysis results.
The lowest plane of the model was considered fixed in the three directions as a boundary condition. Linear static analyses were performed on a personal computer (Intel Core i7 processor, 2.4 GHz, 6.0 GB RAM), using commercial multipurpose finite element software package (ANSYS version 16.0).

\section{Results}

Twelve linear static analyses were performed to evaluate total deformation and Von Mises stress on all model components, as presented in - Fig. 3. The results of tooth structure, bone (cortical and spongy), cement layer and stainless-steel crown were compared with extract findings. Whatever the cement type is, locations of extreme stresses and deformation did not change, which is under loading points and crest of cortical bone, while stresses and deformations patterns changed with load directions.

Total deformation and directional ones did not show significant change with changing cement types on bone. All deformations were found within the acceptable physiological limits. In - Figs. 4 and 5, maximum values of Von Mises stress on tooth structure and crown body were compared. Crown body received increasing stresses by reducing the used cement stiffness, while the tooth structure stresses were decreased by decreasing cement stiffness. These trends were recorded to be from $1 \%$ to $30 \%$ for crown body, and $5 \%$ to $20 \%$ for tooth structure. Increasing load inclination angle reduced the crown stresses difference between the different cement types, while an opposite effect was recorded on tooth structure.

\section{Discussions}

Crowns placed by the Hall technique are strongly supported by promising results, showing high acceptability, longevity, and low-failure rates when compared with conventional treatment options. However, it causes premature contact and increases the occlusal vertical dimension. 
A
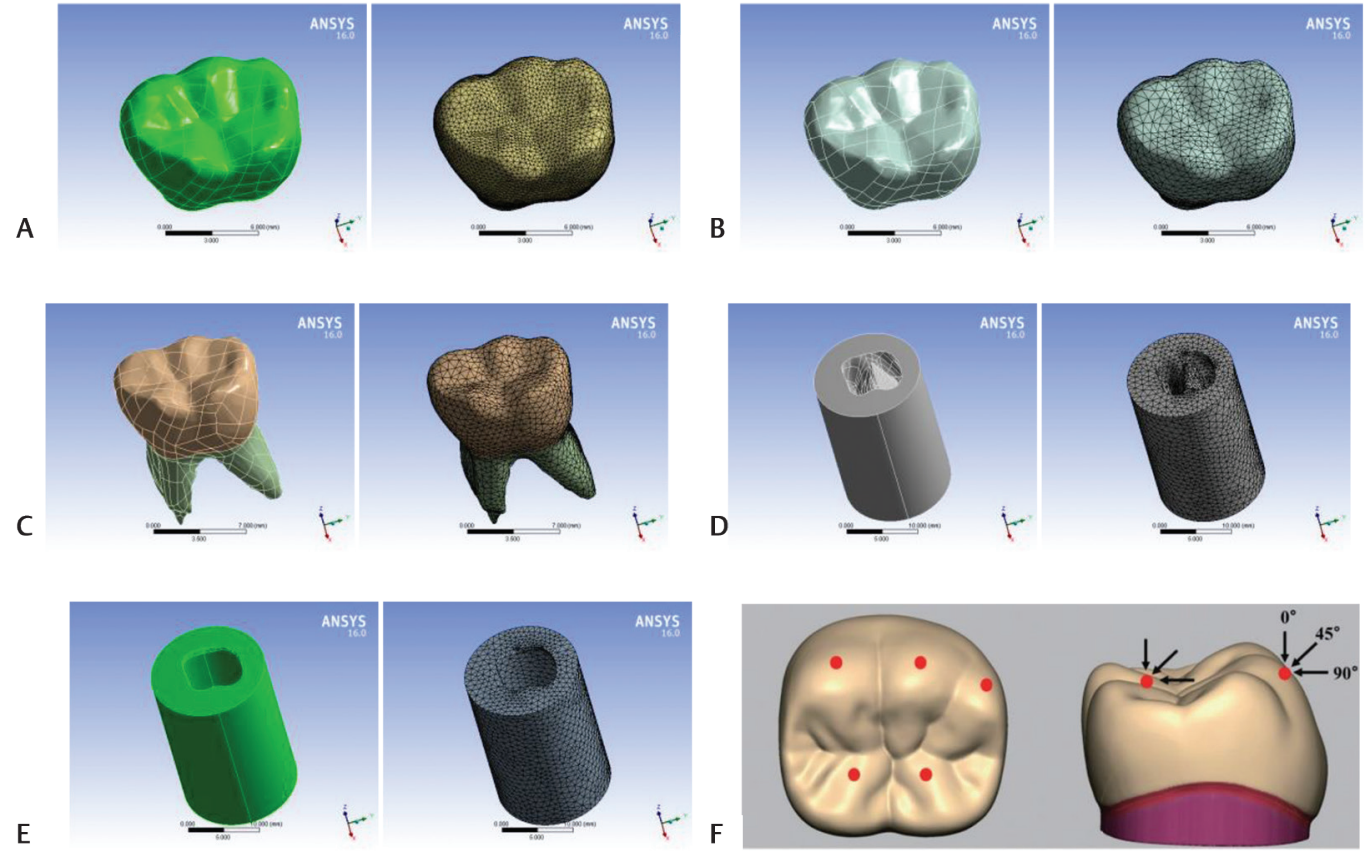

Fig. 2 Screenshots for model components and its mesh (A) crown, (B) cement layer, (C) tooth structure, (D) spongy bone, (E) cortical bone, (F) loading points. ${ }^{22}$

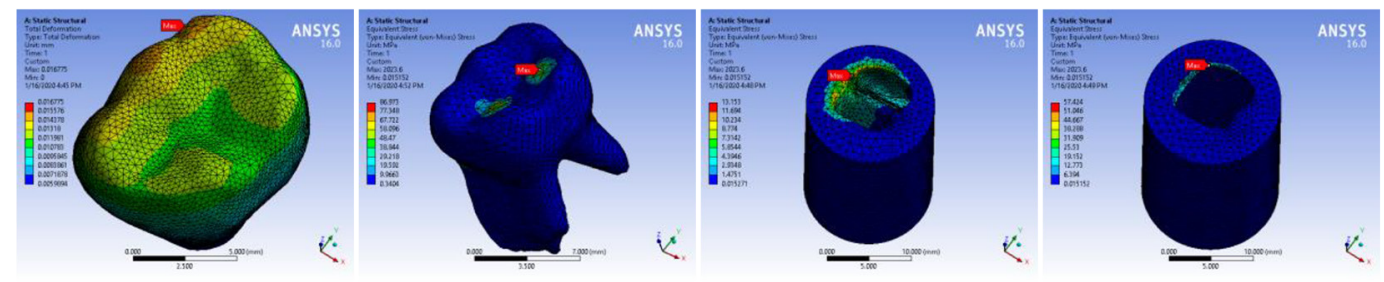

Fig. 3 Sample screenshots for all components.
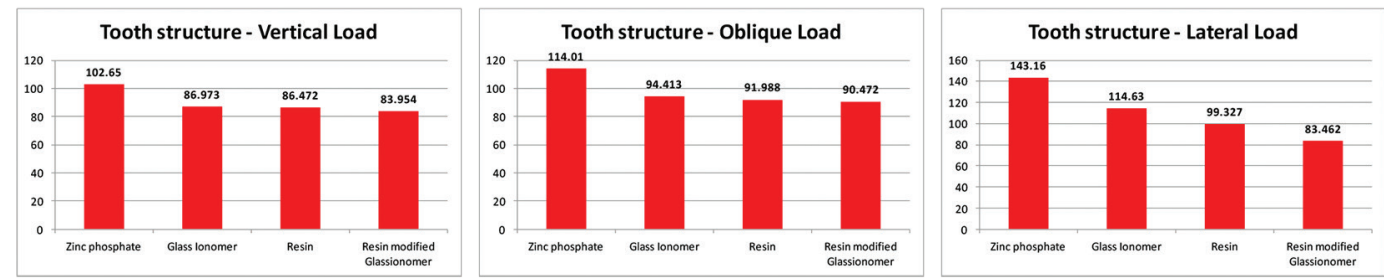

Fig. 4 Tooth structure maximum Von Mises stress comparison.
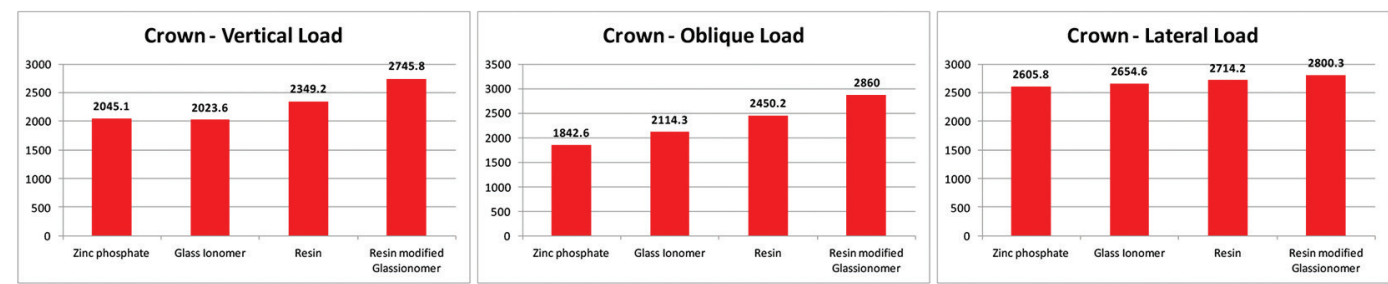

Fig. 5 Crown maximum Von Mises stress comparison. 
Studying the pattern of forces applied to SSC and their effect on both tooth structure and underlying bone may enlighten clinicians with regard to a strategic selection of technique and cementing materials. ${ }^{5}$ Extreme stresses and deformations appeared on crown body appeared under loading points.

The cycle of fatigue of metals occurs, whether it continuously strained or swinging, causing breakdown or deformation. This maximum strain which can be tolerated by the metal until deformation is the compressive stress of metals. The average chewing force of children aged between 5 and 10 years is $330 \mathrm{~N}$, according to several findings. While SSC is a rather durable restoration for children's deciduous teeth, it may be weakened and deformed in long-term usage, and if exposed to excessive strong chewing forces beyond normal chewing power. This force can wear and penetrate the occlusal surface of the crown, which is the key cause of perforation and failure. ${ }^{23,24}$

Although the enamel portion of the tooth carries the major portion of the load, some of these forces are transferred to the periodontium and can induce bone and connective tissue changes. ${ }^{9}$

The second lower primary molar was selected in this study as the mandibular teeth are more susceptible to caries more than the maxillary teeth (mainly the second primary molar) of both the sexes; therefore, it is the most common molar receiving the crown. ${ }^{25}$

The FEM helps in understanding the characteristics of the individual prosthetic components to select optimal design in order to offer the best performance. ${ }^{18}$

As it is impossible to measure the effect of masticatory force applied on dental restorative materials like restorations, crowns, implant, and removable appliances on human living tissue, therefore FEM was used in the present study.

Compared with actual model research, FEM has various advantages. No ethical considerations are necessary, and the nature of the studies can be changed and adjusted as needed. The tests can be replicated and can also be used before surgery or restoration; stress on the bone, dental implants and restoration components should be analyzed and information provided. By using FEM, the researcher can conduct many simulations without needing patients or carrying out human experiments, which is an effective way to improve load management in order to reduce discomfort for patients and achieve long-term clinical performance. $^{12-15,26-28}$

The analysis of finite elements can be performed to analyze a single variable in a complex system, for example, stress on a dental model. The potential of finite element analysis to reliably estimate dental prosthesis is directly related to the manner in which the finite element model is constructed. Finite element solution of physical phenomena governing equations is considered an essential step in many case studies. It improves the understanding of the effect of many oral/ dental devices and materials. ${ }^{29}$

Metal thickness greatly influences cracking strength and thus fatigue, deformation, and fracturing activity. The critical stress factor decreases as the thickness of the material is decreased. The thinner the sample, the more the plastic strain. High-plastic strain causes the fatigue and deformation of the thin samples to grow. ${ }^{30}$

SSC body is thin enough $(0.11-0.17 \mathrm{~mm})$ to be dependent on its support on cement type and thickness. Thus, increasing supporting cement stiffness (elasticity) can reduce the crown stresses and deformations. It may be referred to as elasticity of the cement layer. ${ }^{31}$ When the crown is loaded, stress is induced inside the crown, which can lead to deformation. Evidence can be gathered on the stress concentration areas along with the study of one single variable in a complex structure through finite element analysis. After the appearance of finite element analysis, it became possible to show the prevalence of stress in every part of the tooth and restoration. Accordingly, several studies were conducted..$^{18}$

Tooth morphology (cervical bulging) and the use of proper luting agents are the primary factors and secondary factors which retain the SSC, respectively. If the main factors are standardized, secondary factors will play an important role in increasing their retention. ${ }^{32}$

Many dental materials have been used for several years in crown cementation. Each of these materials have advantages and disadvantages in specific situations. Higher strength cements improve stability by offering a solid base of protection against the powers applied. Zinc phosphate, glass ionomer cement (GIC), resin cement and resin-modified glass ionomer cement (RMGIC) were used in this study, as these are the widely used luting cements for cementing stainless steel crowns.

Zinc phosphate is the earliest luting cement typically used to cement SSC. It is usually considered satisfactory for crown retention, given its strictly mechanical retentive characteristics. It is brittle, has relatively high-mouth solubility, and does not stick to the surface of the tooth. They have a high-degree of compression. ${ }^{33}$

In this study, zinc phosphate showed the best supporting cement from crown point of view, which can be explained as it has the highest mechanical properties including compressive strength and modulus of elasticity. This is comparable with a previous study which confirmed that the restoration-supporting cement modulus of elasticity partly defines the amount of deflection that can occur at the cement-restore interface. Lower elasticity modulus values will result in higher deflection at the interface and higher tensile stresses, which can cause cement failure or even restoration. ${ }^{34}$ In addition, the material's characteristic relies directly on the elastic module of the supporting substructure and the properties of the luting agents, while increasing the thickness and elastic module (rigidity or stiffness) of the substructure contributes to an increase in the flexural strength of the final restoration..$^{35}$

Resin cement is presented in the Hall technique as the best solution to minimize microleakage followed by RMGIC and finally glass ionomer cement. The effectiveness of various cements in terms of the inhibition potential for demineralization could be summarized as: GIC > RMGIC > resin cement ${ }^{36}$ However, Yilmaz et al ${ }^{37}$ claimed that SSC cemented with RMGIC had comparable clinical success levels to glass ionomer luting cement. 
As the main function of luting cement is crown retention, it also transfers load to the supporting tooth structure, acting as shock absorber in addition to creating an integral object that acts as a single part under occlusion force. In this study, it was observed that tooth structure stresses were decreased by decreasing cement stiffness, which explains why RMGIC showed the best performance from tooth structure point of view, as it has the lowest Young's modulus and displayed lower elastic moduli than other types of cement. ${ }^{38}$

In the present study, bone was insensitive to the cement type; locations of extreme stresses and deformation did not change at the crest of cortical bone. Stresses and deformation patterns changed with load directions. This aggregated with another study which tested the effect of luting cement type and thickness on stress distribution within bone concluded that the cement type did not affect the cortical bone, but the cement thickness was further impacted. On the other hand, neither cement type nor thickness affect spongy bone. ${ }^{39}$ These results are in agreement with other studies which have shown that a huge part of occlusal force is transmitted to compact bone while have very few or no effects on cancellous bone. ${ }^{18}$

\section{Conclusions}

The following finding can be extracted from this limited study:

It is recommended to fit stainless-steel crown on hard cement to support and reduce the crown body stresses and deformations. Zinc phosphate showed the best supporting cement from crown point of view.

Using stiffer cement material increases tooth structure stresses, thus, RMGIC showed the best performance from tooth structure point of view.

Bone was nearly insensitive to changing cement type.

\section{Conflict of Interest}

None declared.

\section{References}

1 Seale NS. The use of stainless steel crowns. Pediatr Dent 2002;24(5):501-505

2 Papathanasiou AG, Curzon ME, Fairpo CG. The influence of restorative material on the survival rate of restorations in primary molars. Pediatr Dent 1994;16(4):282-288

3 Zinelis S, Lambrinaki T, Kavvadia K, Papagiannoulis L. Morphological and compositional alterations of in vivo aged prefabricated pediatric metal crowns (PMCs) Dent Mater 2008;24(2):216-220

4 Innes N, Evans D, Stewart M, Keightley A. The Hall technique: a minimal intervention, child centred approach to managing the carious primary molar. University of Dundee. Available at: https://dentistry.dundee.ac.uk/files/3M_93C\%20 HallTechGuide2191110.pdf https://upload.wikimedia.org/ wikipedia/commons/9/91/HallTechGuide_V4.pdf. Accessed July 19, 2020

5 Altoukhi DH, El-Housseiny AA. Hall technique for carious primary molars: a review of the literature. Dent J (Basel) 2020;8(1):1-13
6 Innes N, Stewart M, Souster G, Evans D. The Hall Technique; retrospective case-note follow-up of 5-year RCT. Br Dent J 2015;219(8):395-400

7 Page LA, Boyd DH, Davidson SE, McKay SK, Thomson WM, Innes NP. Acceptability of the Hall technique to parents and children. N Z Dent J 2014;110(1):12-17

8 Abu Serdaneh S, AlHalabi M, Kowash M, et al. Hall technique crowns and children's masseter muscle activity: A surface electromyography pilot study. Int J Paediatr Dent 2020;30(3):303-313

9 Singh DK, Jalaluddin M, Rajeev R. Trauma from occlusion: the overstrain of the supporting structures of the teeth. Indian J Dent Sci 2017;9:126-132

10 Subramaniam P, Kondae S, Gupta KK. Retentive strength of luting cements for stainless steel crowns: an in vitro study. J Clin Pediatr Dent 2010;34(4):309-312

11 Geramy A, Sharafoddin F. Abfraction: 3D analysis by means of the finite element method. Quintessence Int 2003;34(7):526-533

12 Lauritano F, Runci M, Cervino G, Fiorillo L, Bramanti E, Cicciù M. Three-dimensional evaluation of different prosthesis retention systems using finite element analysis and the Von Mises stress test. Minerva Stomatol 2016;65(6):353-367

13 Cervino G, Fiorillo L, Arzukanyan AV, Spagnuolo G, Campagna P, Cicciù M. Application of bioengineering devices for stress evaluation in dentistry: the last 10 years FEM parametric analysis of outcomes and current trends. Minerva Stomatol 2020;69(1):55-62

14 Cicciù M, Cervino G, Milone D, Risitano G. FEM Analysis of Dental Implant-Abutment Interface Overdenture Components and Parametric Evaluation of Equator ${ }^{\circledR}$ and Locator ${ }^{\circledR P r o s t h o d o n t i c s}$ Attachments. Materials (Basel) 2019;12(4):592

15 Cicciù M, Cervino G, Milone D, Risitano G. FEM investigation of the stress distribution over mandibular bone due to screwed overdenture positioned on dental implants. Materials (Basel) 2018;11(9):1512

16 Marlière DAA, Demètrio MS, Picinini LS, Oliveira RG, Netto HDMC. Accuracy of computer-guided surgery for dental implant placement in fully edentulous patients: A systematic review. Eur J Dent 2018;12(1):153-160

17 Hakim MAA, Khatab NMA, Mohamed KMG, Elheeny AA. A comparative three-dimensional finite element study of two space regainers in the mixed dentition stage. Eur J Dent 2020;14(1):107-114

18 Al Qahtani WMS, Yousief SA, El-Anwar MI. Recent advances in material and geometrical modelling in dental applications. Open Access Maced J Med Sci 2018;6(6):1138-1144

19 Sabit AM, Mohsen CA, El-Anwar MI, Metawally MM. An in-vitro study of crowned endodontically treated immature permanent central incisor reinforced with different types of aesthetic posts using FEA. IOSR Journal of Dental and Medical Sciences 2017;16(8):82-87

20 Fathy SM, El-Anwar MI, El-Fallal AA, El-Negoly SA. Threedimensional finite element analysis of lower molar tooth restored with fully milled and layered zirconia crowns. J Dent Health Oral Disord Ther 2014;1(4):00022

21 Romeed SA, Dunne SM. Stress analysis of different post-luting systems: a three-dimensional finite element analysis. Aust Dent J 2013;58(1):82-88

22 Ha S-R, Kim S-H, Han J-S, et al. The influence of various core designs on stress distribution in the veneered zirconia crown: a finite element analysis study. J Adv Prosthodont 2013;5(2):187-197

23 Yilmaz Y, Kara NB, Yilmaz A, Sahin H. Wear and repair of stainless steel crowns. Eur J Paediatr Dent 2011;12(1):25-30 
24 Maruyama N, Mori D, Hiromoto S, Kanazawa K, Nakamura M. Fatigue strength of 316L-type stainless steel in simulated body fluids. Corros Sci 2011;53(6):2222-2227

25 Sachdeva A, Punhani N, Bala M, Arora S, Gill GS, Dewan N. The prevalence and pattern of cavitated carious lesions in primary dentition among children under 5 years age in Sirsa, Haryana (India) J Int Soc Prev Community Dent 2015;5(6):494-498

26 Bramanti E, Cervino G, Lauritano F, et al. FEM and von mises analysis on prosthetic crowns structural elements: Evaluation of different applied materials. Scientific World 2017;2017:1029574

27 Cicciù M, Bramanti E, Cecchetti F, Scappaticci L, Guglielmino E, Risitano G. FEM and Von Mises analyses of different dental implant shapes for masticatory loading distribution. Oral Implantol (Rome) 2014;7(1):1-10

28 Cicciù M, Risitano G, Maiorana C, Franceschini G. Parametric analysis of the strength in the "Toronto" osseous-prosthesis system. Minerva Stomatol 2009;58(1-2):9-23

29 Al Qahtani WMS, El-Anwar MI. Advanced computational methods in Bio-Mechanics. Open Access Maced J Med Sci 2018;6(4):742-746

30 Dowling NE, Mechanical Behavior of Materials, Engineering Methods for Deformation, Fracture and Fatigue. 3rd ed Prentice-Hall; 2007

31 May LG, Kelly JR, Bottino MA, Hill T. Effects of cement thickness and bonding on the failure loads of CAD/CAM ceramic crowns: multi-physics FEA modeling and monotonic testing. Dent Mater 2012;28(8):e99-e109
32 Myers DR, Bell RA, Barenie JT. The effect of cement type and tooth preparation on the retention of stainless steel crowns. J Pedod 1981;5(4):275-280

33 Wilson AD, Zinc oxide dental cements in scientific aspects of dental materials. In: Von Fraunhofer JA, ed 1st ed London: Butterworth's; 1975305

34 Craig RG, Farah JW, Powers JM. Modulus of elasticity and strength properties of dental cements. J Am Dent Assoc 1976;92(3):588-591 doi:10.14219/jada.archive.1976.0571

35 Campbell SD. A comparative strength study of metal ceramic and all-ceramic esthetic materials: modulus of rupture. J Prosthet Dent 1989;62(4):476-479

36 Panthri P, Srivastava N, Rana V. How efficacious are stainless steel crown luting cements: an ex vivo comparative study. EC Dental Science 2018;17(11):1887-1898

37 Yilmaz Y, Simsek S, Dalmis A, Gurbuz T, Kocogullari ME. Evaluation of stainless steel crowns cemented with glass-ionomer and resin-modified glass-ionomer luting cements. Am J Dent 2006;19(2):106-110

38 Li ZC, White SN. Mechanical properties of dental luting cements. J Prosthet Dent 1999;81(5):597-609

39 El-Anwar MI, Tamam RA, Fawzy UM, Yousief SA. The effect of luting cement type and thickness on stress distribution in upper premolar implant restored with metal ceramic crowns. Tanta Dent J 2015;12(1):48-55 